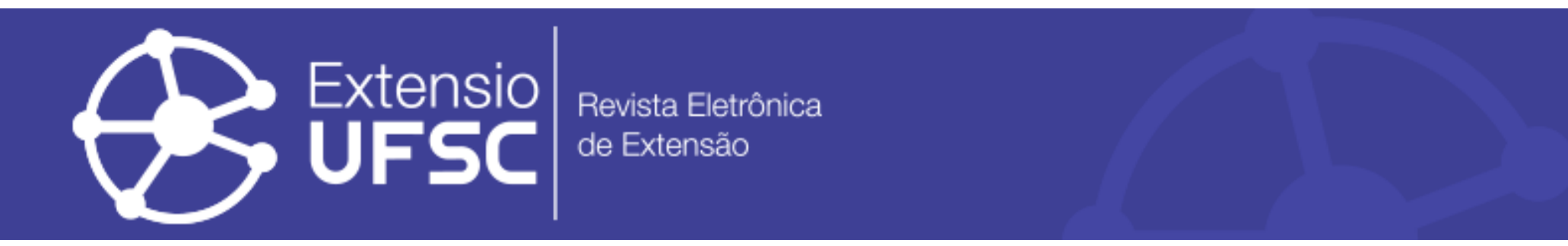

\title{
A EDUCAÇÃO EM SAÚDE NO AMBIENTE HOSPITALAR: RELATO DE EXPERIÊNCIA SOBRE PREVENÇÃO DE LESÕES POR PRESSÃO
}

\author{
Bruna Stamm \\ Universidade Federal do Pampa \\ bruna-stamm@hotmail.com
}

\author{
Carlos Eduardo Messa Ponse \\ Universidade Federal do Pampa \\ carloseduardomp94@hotmail.com
}

Karine Matos dos Santos

Universidade Federal do Pampa

karine.ms@hotmail.com.br

\section{Resumo}

O objetivo deste é compartilhar a experiência de discentes de enfermagem em atividade educativa sobre Lesões por Pressão com familiares/acompanhantes de pacientes hospitalizados. Trata-se de um relato de experiência, desenvolvido a partir de vivências dos discentes de graduação em enfermagem da Universidade Federal do Pampa, durante as atividades práticas na clínica médica de um hospital de grande porte de uma cidade da fronteira oeste do Rio Grande do Sul/ Brasil, desenvolvidas em 2016. Para a atividade de educação em saúde sobre manejo e medidas preventivas de lesões de pressão, foram reunidas cinco acompanhantes/familiares das pacientes hospitalizadas, nas quais todas apresentavam Lesão por Pressão de graus variados. A roda de conversa teve duração de uma hora, e envolveu apresentação de cartazes com os temas: graus, localização e coberturas das lesões. A vivência proporcionou aprofundar conhecimentos no contexto de leões, permitiu uma melhor assistência aos pacientes e auxiliou familiares/cuidadores visando um melhor desfecho no cuidado as lesões por pressão.

Palavras-chave: Lesão por Pressão. Enfermagem. Educação em Saúde. Hospital.

\section{HEALTH EDUCATION IN THE HOSPITAL ENVIRONMENT: EXPERIENCE REPORT ON PREVENTION OF PRESSURE INJURY}

\begin{abstract}
The aim of this study is to share the experience of nursing students in educational activity on Pressure Injury with family members / companions of hospitalized patients. This is an experience report, developed from the experiences of graduate students in nursing at the Federal University of Pampa, during the practical activities in the medical clinic of a large hospital in a city on the western border of Rio Grande do Sul/Brazil, developed in 2016. For the health education activity on management and preventive measures of pressure injuries, five companions/relatives of the hospitalized patients were assembled, in which all presented Pressure Injury of varying degrees. The talk wheel lasted one hour, and involved the presentation of posters with the themes: degrees, location and coverage of the lesions. The experience provided a deepening of knowledge in the context of lions, allowed a better assistance to patients and assisted family/caregivers aiming for a better outcome in the care of pressure injuries.
\end{abstract}

Keywords: Pressure Ulcer. Nursing. Health Education. Hospital.

\section{LA EDUCACIÓN EN SALUD EN EL MEDIO AMBIENTE HOSPITALARIO: RELATO DE EXPERIENCIA SOBRE PREVENCIÓN DE LESIONES POR PRESIÓN}

\section{Resumen}

El objetivo de este es compartir la experiencia de discentes de enfermería en actividad educativa sobre Lesiones por Presión con familiares/acompañantes de pacientes hospitalizados. Se trata de un relato de experiencia, desarrollado a partir de vivencias de los discentes de graduación en enfermería de la Universidad Federal del Pampa, durante las actividades prácticas en la clínica médica de un hospital de gran porte de una ciudad de la frontera oeste de Rio Grande do Sul En el presente trabajo se analizaron los resultados obtenidos en el análisis de los resultados obtenidos en el estudio. La rueda de conversación tuvo una duración de una hora, e implicó presentación de carteles con los temas: grados, ubicación y coberturas de las lesiones. La vivencia proporcionó profundizar conocimientos en el contexto de leones, permitió una mejor asistencia a los pacientes y ayudó a familiares/cuidadores buscando un mejor desenlace en el cuidado de las lesiones por presión.

Palavras clave: Úlcera por Presión. Enfermeria. Educación en Salud. Hospital. 
A educação em saúde no ambiente hospitalar: relato de experiência sobre prevenção de lesões por pressão

\section{INTRODUÇÃO}

Os pacientes hospitalizados com problemas de mobilidade apresentam dificuldades de alternância de decúbito e requerem manejo adequado quanto ao reposicionamento no leito (MADO LIVRAMENTO NEVES SILVA et al., 2013). As Lesões por Pressão (LP) podem agravar o quadro clínico dos pacientes e aumentar o tempo de internação, sendo necessário investigar os fatores associados ao desenvolvimento destas lesões (PEREIRA et al., 2017). A mudança de posicionamento é a principal intervenção terapêutica na prevenção de LP (CALIRI et al., 2016).

Em abril de 2016, o órgão americano National Pressure Ulcer Advisory Panel (NPUAP) substituiu o termo "Úlcera” por "Lesão por Pressão". Diferentemente de boa parte das alterações de pele, a LP tem sido fonte de preocupação para os serviços de saúde, pois existem altos índices, principalmente em populações de risco, o que representa um desafio aos profissionais de saúde na implementação de ações preventivas e de utilização dos avanços tecnológicos para o tratamento (MARTIN et al., 2017; ROGENSKI, KURCGANT, 2016).

A LP é um dano localizado na pele e/ou nos tecidos moles subjacentes, geralmente ocorre sobre uma proeminência óssea ou está relacionada ao uso de dispositivo médico ou de outro artefato (CALIRI et al., 2016).

Dentre alguns dos fatores de risco que podem desenvolver LP estão a Hipertensão Arterial Sistêmica (HAS), o diabetes, a inconsciência, a imobilização, a perda de sensibilidade, de função motora, de continência urinária ou fecal, a presença de espasmos musculares, as deficiências nutricionais, as anemias, o índice de massa corporal (muito alto ou muito baixo), as doenças circulatórias, a doença arterial periférica, a imunodeficiência ou uso de corticosteroide, e o tabagismo (MORAES et al., 2016).

A prevenção das LP deve ser o foco dos profissionais responsáveis pelo cuidado do paciente. A abordagem preventiva deve ser multidisciplinar e tem início na identificação precoce dos pacientes suscetíveis, devendo abranger a equipe cuidadora, além dos familiares envolvidos e do próprio paciente, quando possível. Mecanismos de distribuição da pressão, mudança periódica de posição, controle da incontinência, cuidados com a pele e nutrição são as principais medidas envolvidas (ASCARI et al., 2014). A inclusão da família nesses cuidados tem sido de suma importância para ajudar no cuidado efetivo da lesão, por exemplo, quando o paciente recebe alta e os cuidados com a lesão passam a ser diários e no domicílio.

Com isso, promover e educar para a saúde são práticas indissociáveis, devendo, dessa maneira, envolver todos os grupos de indivíduos nesse processo. O conceito de educação em 
A educação em saúde no ambiente hospitalar: relato de experiência sobre prevenção de lesões por pressão

saúde se associa ao conceito de promoção à saúde, uma vez que ambas tratam dos processos que integram a participação da população em seu contexto cotidiano, e não apenas pessoas com algum adoecimento (MACHADO et al., 2007).

O enfermeiro é um agente fundamental na educação em saúde, pois pode compreender as necessidades dos pacientes, evitando o aumento de LP e assim, proporcionar uma qualidade de vida. Destaca-se fundamental importância da atuação da equipe multiprofissional para a prevenção da saúde, para dar continuidade no cuidado pós-alta, realizar as conexões de redes no domicílio, para o acompanhamento no tratamento e cuidado com LP.

O objetivo deste é compartilhar a experiência de discentes de enfermagem em uma atividade educativa sobre LP com familiares/acompanhantes de pacientes hospitalizados.

\section{MATERIAIS E MÉTODOS}

O estudo trata-se de um relato de experiência, desenvolvido a partir de vivências de discentes do $4^{\circ}$ semestre de graduação em enfermagem, durante as atividades práticas do componente curricular de Semiotécnica em Enfermagem da Universidade Federal do Pampa (UNIPAMPA).

A vivência ocorreu na clínica médica de um hospital de grande porte de uma cidade da fronteira oeste do Rio Grande do Sul/ Brasil, sob supervisão direta de uma enfermeira-docente. A capacidade instalada do referido hospital é de 199 leitos, referente às internações pediátrica, neonatal, obstetrícia, clínica, cirúrgica, psiquiátrica, Unidade de Terapia Infantil e Adulta. Atualmente assiste à população com cobertura do Sistema Único de Saúde (SUS) que corresponde a uma média mensal de 650 atendimentos, e também outros convênios de saúde.

O corpo clínico é composto por aproximadamente 100 médicos de várias especialidades, sendo que 58 médicos fazem parte do quadro funcional do hospital, que conta com 38 enfermeiros, 40 auxiliares de enfermagem, 174 técnicos em enfermagem, 1 nutricionista, 04 farmacêuticos bioquímicos e mais 216 funcionários do serviço de apoio e administração, sendo referência regional em oncologia e neurocirurgia para mais de 30 municípios do Rio Grande do Sul/Brasil.

A unidade de clínica médica contém 47 leitos, distribuídos em uma enfermaria feminina, masculina e de saúde mental, e seis leitos semi-privativos. Quanto ao perfil dos pacientes hospitalizados na unidade incluem, prioritariamente, idosos com doenças respiratórias, endócrinas e oncológicas. A equipe de saúde da unidade é composta por um enfermeiro (a) por jornada de trabalho, quatro técnicos de enfermagem, um maqueiro e uma secretária. 
A educação em saúde no ambiente hospitalar: relato de experiência sobre prevenção de lesões por pressão

As atividades práticas do componente curricular foram desenvolvidas no segundo semestre do ano de 2016, em grupos com cinco acadêmicos de enfermagem e a docente responsável. As mesmas tiveram por finalidade promover o cuidado ao paciente nas necessidades de higiene e conforto, térmicas e vasculares, oxigenação, hidratação e nutrição, eliminação urinária e intestinal, integridade cutâneo das mucosas e administração de medicamentos. Foram realizadas durante nove dias de atividades práticas, nas segundas e terças-feiras, no turno matutino.

O grupo de prática foi dividido conforme a demanda da unidade, no qual tiveram a oportunidade de cuidar de pacientes com diversas patologias, como tais: HAS, Diabetes Melittus (DM), incontinência urinária, amigdalite, doenças crônicas e respiratórias. O objetivo dessa prática era desenvolver competências e habilidades dos procedimentos técnicos de enfermagem, como cateterismo sonda nasogástrica e nasoentérica, punção venosa, instalação de O2, instalação de dietas, administração de medicações e curativos.

Também foi vivenciado situações com pacientes em fase terminal, óbitos e parada cardiorrespiratória. Foi a partir dessas atividades práticas do componente curricular que se desenvolveu uma ação de educação em saúde sobre LP com familiares/acompanhantes de pacientes femininas hospitalizadas na referida unidade.

Para a atividade de educação em saúde sobre manejo e medidas preventivas de LP, foram reunidas em uma das enfermarias femininas da unidade, as cinco acompanhantes/familiares das pacientes hospitalizadas, nas quais todas apresentavam LP de graus variados. A atividade deu-se através de uma roda de conversa, com duração de uma hora, e envolveu apresentação de cartazes com os temas: graus, localização e coberturas das lesões; desenvolvida pelos acadêmicos, juntamente com um técnico administrativo em educação que acompanhava as atividades práticas.

\section{RESULTADOS E ANÁLISES}

Conforme o desenvolvimento das atividades práticas, o grupo acadêmico identificou alto índice de pacientes com LP, e que muitos apresentavam fatores de risco para tal. Em umas das discussões dos casos clínicos e de cuidados de alguns pacientes, notou-se a preocupação e a necessidade de ações para evitar o aumento das LP e preveni-las. Em uma das conversas com a docente, surgiu a idéia dos acadêmicos realizarem uma atividade de educação em saúde sobre LP e pensando no cuidado com pacientes acamados ou dependentes de cuidado na unidade hospitalar. 
A educação em saúde no ambiente hospitalar: relato de experiência sobre prevenção de lesões por pressão

Inicialmente ocorreu uma reunião breve com a docente e a enfermeira responsável da unidade para destacar a necessidade da ação, e que uma atividade como a de educação em saúde contribuiria tanto para o processo ensino-aprendizado dos acadêmicos, para a equipe de saúde como para os próprios familiares/acompanhantes.

Nesta interlocução, a educação em saúde é caracterizada como um instrumento transformador, uma vez que possibilita uma melhoria na qualidade de vida dos grupos e pessoas envolvidas. A promoção da saúde por meio de ações educativas gera responsabilidade para os indivíduos atuarem diante de suas próprias vidas corroborando com a qualidade de vida (SOUZA et al., 2005).

Após, levantou-se fomentos teóricos, com revisões bibliográficas através de livros, buscas em periódicos e imagens demonstrativas, tendo como foco principal a implementação de relógios de alternância de decúbito, fixados nas cabeceiras dos leitos para melhor compreensão deste cuidado para os acompanhantes/familiares e equipe de enfermagem. Esta estratégia continha informações de fácil entendimento, com horários e posições para a alternância de decúbito, demonstradas com imagens no relógio, fixadas em todos os leitos da unidade.

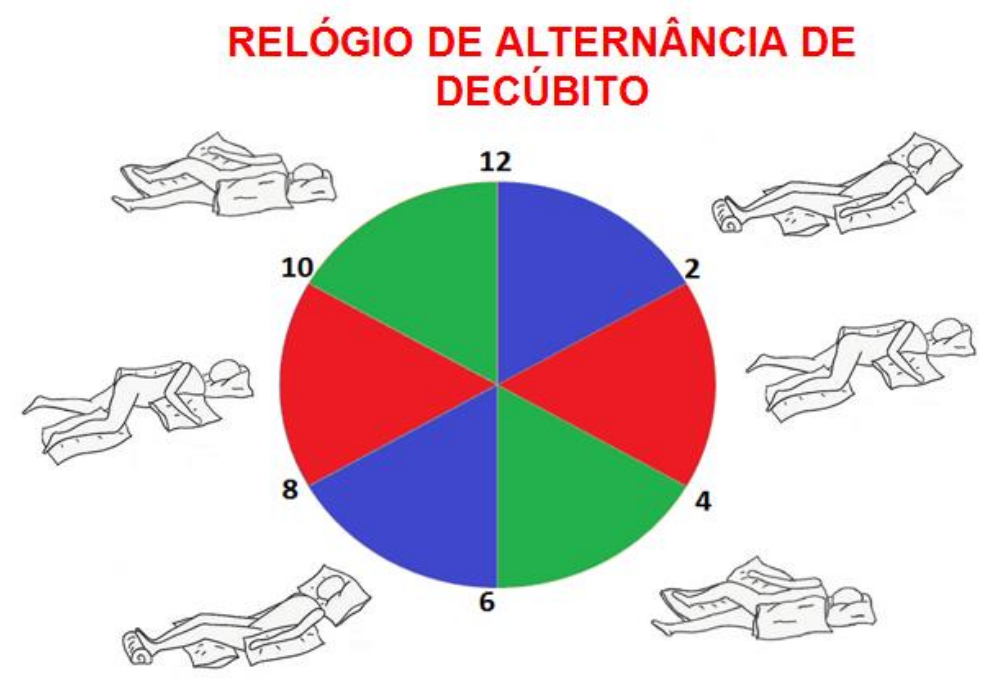

Ilustração 1 - Relógio de alternância de decúbito confeccionado para a atividade de educação em saúde. Fonte: Elaboração dos autores.

A roda de conversa com os familiares/acompanhantes das pacientes hospitalizadas foi realizada pelo grupo de acadêmicos, no qual visaram orientar e esclarecer sobre as principais medidas preventivas e de manejo das LP. Durante aproximadamente uma hora, foi possível que os familiares/acompanhantes compartilhassem suas vivências e experiências, seus medos, suas dificuldades, as dúvidas e ações estratégicas com o cuidado das pacientes com LP. Muitas orientações foram suscitadas sobre reposicionamento no leito, suas implicações e cuidados pós- 
A educação em saúde no ambiente hospitalar: relato de experiência sobre prevenção de lesões por pressão

alta. Importante destacar que a atividade promove um espaço de fortalecimento do conhecimento dos envolvidos, bem como pode repercutir positivamente na qualidade o cuidado prestado pelos acompanhantes/familiares às pacientes, e assim, impactar nas ações, na comunicação e relação da equipe de enfermagem para com estes.

Em relação ao processo formativo em enfermagem, tem sido necessária uma formação com conhecimentos, habilidades e atitudes necessárias como diferencial para a prestação de um cuidado integral. Neste constructo, um estudo descreveu a construção de cenário clínico simulado de alta fidelidade destinado ao ensino aprendizagem da assistência de enfermagem ao paciente com LP, e destacam, dentre outros, que tais recursos educativos auxiliam o processo de ensino aprendizagem e a atualização dos profissionais em prevenção e tratamento de lesões (MAZZO et al., 2018).

$\mathrm{Na}$ atividade, inicialmente os familiares/acompanhantes foram indagando sobre quais os conhecimentos prévios que possuíam sobre as causas das LP. Por conseguinte, iniciou-se uma apresentação sucinta sobre a nomenclatura, seu desenvolvimento, aspectos fisiológicos envolvidos, tipos de tecidos, grau das lesões e quais tecidos eram afetados. Isto foi apresentado por meio de figuras imprensas e explanação verbal dos acadêmicos.

Um estudo identificou o conhecimento dos familiares de pessoas internadas com LP acerca de seus direitos à reparação de danos causados pela lesão, e para os familiares, as pessoas que se encontram hospitalizadas inevitavelmente desenvolvem as LP devido à imobilização e umidade. Porém, estas famílias desconheciam os fatores que levavam seus entes a desenvolverem LP, o que limita sua participação no cuidado de prevenção e tende a persistir quando retornarem aos seus domicílios (OLIVEIRA et al., 2014).

A importância da educação em saúde para famílias deve se iniciar desde o momento da internação hospitalar, para facilitar o despertar do interesse dos mesmos para realizar o cuidado em casa, saber quem recorrer, redes a serem procuradas, e também para esclarecer o entendimento dos procedimentos e cuidados prestados ao paciente.

Um dos tópicos da atividade de educação em saúde foi a realização dos curativos e dos tipos de coberturas típicas adequadas para LP. Utilizou-se de figuras que apresentavam as localizações frequentes para o aparecimento das lesões. Ao decorrer da atividade, os familiares/acompanhantes relataram suas dúvidas, tais como, posições adequadas, uso de coberturas, inseguranças com o manejo com os pacientes, risco de causar novas lesões e o aumento das mesmas, a partir da temática introduzida pelo grupo. Foi possível esclarecer ao grupo algumas condutas quanto ao atendimento inicial, manejo com as lesões e medidas de prevenção. 
A educação em saúde no ambiente hospitalar: relato de experiência sobre prevenção de lesões por pressão

No que se refere aos recursos utilizados para o tratamento de LP, curativos com diferentes tipos de coberturas são considerados a terapia convencional, uma vez que estimulam o processo cicatrizal a médio e longo prazo, dependendo do estadiamento, e com base no grau de destruição tecidual das lesões e das condições do paciente (PALAGI et al., 2015).

Um estudo atual compreendeu a perspectiva do enfermeiro frente aos cuidados de enfermagem no tratamento de feridas em ambiente hospitalar de ensino, e destacou que existe um consenso sobre a importância do trabalho do enfermeiro, principalmente inserido em equipe multidisciplinar, para efetividade no tratamento das feridas. $\mathrm{O}(\mathrm{a})$ enfermeiro(a) atua como figura chave no processo de prevenção ou tratamento de lesões, assim como na construção de protocolos clínicos e treinamentos, para qualificação da assistência (MACHADO et al., 2017).

Outro tópico elucidado foi sobre a alternância de decúbito e reposicionamento do paciente no leito. Foi demostrado em uma paciente hospitalizada qual a forma mais adequada do manejo no leito, tendo envolvimento dos familiares/acompanhantes, estimulando-os e empoderando-os com essa prática. Ao final, realizou-se a fixação dos relógios de alternância de decúbitos na parede em cima do leito de cada paciente para facilitar a visualização, e estes foram orientados sobre a necessidade de alternância de decúbito de duas em duas horas para a prevenção de LP.

Os acompanhantes/familiares se demonstraram interessados em executar com seus familiares os cuidados ensinados, como o posicionamento adequado e seguro, que no início evidenciaram não saber corretamente, sendo assim alcançados nossos resultados esperados, visto que houve dedicação e participação em todas as atividades propostas.

Após, o grupo reuniu-se com a equipe para feedback da atividade. A estratégia do relógio nas paredes foi uma opção econômica e viável para a prevenção de LP.

\section{CONSIDERAÇÕES FINAIS}

A vivência proporcionou aprofundar conhecimentos no contexto de LP, permitiu uma melhor assistência aos pacientes e auxiliou familiares/cuidadores visando um melhor desfecho no cuidado aos seus entes, com vistas no manejo no domicílio destas famílias. Atividades educativas em saúde no âmbito hospitalar são necessárias e viáveis na assistência as LP, e incluir a família como elemento estratégico do cuidado aos pacientes deve ser uma ação indispensável das equipes de saúde. 
A educação em saúde no ambiente hospitalar: relato de experiência sobre prevenção de lesões por pressão

\section{REFERÊNCIAS}

ASCARI, Rosana Amora et al. Úlcera por pressão: um desafio para a enfermagem. Brazilian Journal of Surgery and Clinical Research, n. 1, v. 6, p. 11-6, 2014.

CALIRI, M. H. L. et al. Classificação das lesões por pressão-consenso NPUAP 2016: adaptada culturalmente para o Brasil. Assoc Bras Estomaterapia-SOBEST e da Assoc Bras Enferm em Dermatologia-SOBENDE, 2016.

MACHADO, Maria de Fátima Antero Sousa et al. Integralidade, formação de saúde, educação em saúde e as propostas do SUS: uma revisão conceitual. Revista Ciência e Saúde Coletiva, v. 12, n. 2, p. 335-342, 2007.

MADO LIVRAMENTO NEVES SILVA, Maria et al. Úlcera por pressão em unidade de terapia intensiva: análise da incidência e lesões instaladas. Revista da Rede de Enfermagem do Nordeste, v. 14, n. 5, p. 938-44, 2013.

MARTIN, Donna et al. Healthy Skin Wins: A Glowing Pressure Ulcer Prevention Program That Can Guide Evidence-Based Practice. Worldviews on Evidence-Based Nursing, v. 14, n. 6, p. 473-483, 2017.

MAZZO, Alessandra et al. Teaching of pressure injury prevention and treatment using simulation. Escola Anna Nery, v. 22, n. 1, p. 1-8, 2018.

MACHADO, Fernanda Sabrina et al. Perspectiva do enfermeiro frente à assistência no tratamento de feridas em ambiente hospitalar. Revista de Epidemiologia e Controle de Infecção, v. 7, n. 3, p. 134-139. 2017.

OLIVEIRA, Danielle et al. Conhecimento de familiares acerca das úlceras por pressão e de seus direitos à reparação. Revista Baiana de Enfermagem, v. 28, n. 1, p. 23-30, 2014.

PALAGI, Sofia et al. Laser therapy in pressure ulcers: evaluation by the Pressure Ulcer Scale for Healing and Nursing Outcomes Classification. Revista da Escola de Enfermagem da USP, v. 49 , n. 5, p. 826-833, 2015.

PEREIRA, Antônio Francisco Machado et al. Pressure injury incidence in a university hospital. Revista de Enfermagem da Universidade Federal do Piauí, v. 6, n. 1, p 36-9, 2017.

ROGENSKI, Noemi Marisa Brunet; KURCGANT, Paulina. The incidence of pressure ulcers after the implementation of a prevention protocol. Revista latino-americana de enfermagem, v. 20, n. 2, p. 333-339, 2012.

SOUZA, Aline Corrêa de et al. A educação em saúde com grupos na comunidade: uma estratégia facilitadora da promoção da saúde. Revista gaúcha de enfermagem, v. 26, n. 2, p. 147-153, 2005.

Recebido em: 03/10/2018

Aceito em: 21/03/2019 